\title{
THE PARASITE OF MALARIA
}

\section{AS OBSERVED IN THE MALARIAL FEVERS OF THE SOUTH OF SPAIN}

\author{
$\mathbf{B Y}$ \\ ROBERT J. MARSHALL, M.B., C.M., \\ AND \\ GEORGE THIN, M.D.
}

Received November 5th, 1895-Read February 11th, 1896

LAVERAN's discovery of the hæmatozoon of malarial fevers has suggested many important problems in connection with the pathology of paludism, amongst which perhaps the most urgent is the question of the existence and distribution of varieties or species of the parasites which are associated with corresponding forms of malarial infections. Of these types, two have been studied and described by Golgi, who has shown that quartan and tertian agues owe their differences to distinctive varieties of the parasite, the parasite of quartan fever requiring three days, and that of the tertian fever two days, for its development. Although Golgi's discoveries have not yet received universal recognition, they have been accepted by the great majority of those who have 
seriously worked at the subject, and they have naturally led to long investigations regarding the nature of the parasites associated with those other types of malarial fever which differ in their course from the classical quartans and tertians. Marchiafara, Celli, Bignami and Bastianelli, Grassi and Feletti, and other Italian observers have recorded many remarkable observations regarding the parasites found in the severe forms of fever observed, more particularly in summer and autumn, in the South of Italy. The fact that the parasites of these fevers are not usually found in the sporulating form in blood taken from the finger has, amongst other causes, increased the difficulty of the study, and although the Italian physicians have succeeded in establishing many important facts in connection with them, there are other points regarding which our knowledge is very defective.

As malarial fevers assume a variety of types in different parts of the world, and as at least three-some authorities would say five-varieties of parasites have already been found associated with as many distinct types of fever, it appears probable that as many varieties of parasites exist as there are varieties of fever, and there is thus opened out before us a large field for observation in undertaking the study of the parasites found in the blood of malarial patients. Before this necessary labour is completed much work must be done and many workers are required.

The following paper is offered by the authors as a contribution to this aspect of the subject. They are not aware that the malarial fevers of Spain have yet received special study; and they make no pretention to anything like an exhaustive study even of these fevers. By a close observation of a small number of selected cases of the fevers of the Province of Huelva they are able to put on record a certain number of facts which they hope may render their contribution, fragmentary though it is, of some value as a basis for more extended investigation.

For the more easy understanding of their record they ask that the following facts may be borne in mind. 
When the young spore or amœba of the hæmatozoon attacks a red corpuscle, it is seen in the fresh blood as a small, clear, colourless object, with or without amœboid movements. As it grows, minute pigment granules are observed in its substance, frequently in active movement, and presumably by their movements indicating currents in the protoplasm of the parasite. The pigment observed in these parasites has characteristic marks in the recognised varieties, its coarseness or fineness and mode of arrangement being fairly uniform for each variety. The pigment therefore becomes useful as a diagnostic index. As the parasite develops, the red corpuscle, in some forms, alters in size and in colour, these changes being characteristic of the variety of the parasite. The reproduction of the parasite is effected by division into segments. The number of segments, the time required for their development, their relation to the size of the corpuscle, and their presence or absence in blood taken from the finger,- their absence in the blood from the finger coinciding with their presence in the blood from the spleen, -afford other important diagnostic marks. In most of, if not all, the varieties of the parasite there is found in the blood from the finger rounded or ovoid bodies of various sizes, penetrated in all directions with pigment granules, and without any trace of spore formation. These are generally recognised as sterile bodies in which reproductive development has not taken place. Small rounded clumps are also found in all the varieties, with adherent portions of pigment. The nature of these objects is not always clear, but in many cases they are undoubtedly from the centre of the sporulating body, the débris left after detachment of the spores. Pigmentbearing leucocytes are also found in malarial fever in blood taken from the finger, and the time after the attack during which they are found has also a special relation to the variety of the parasite, affording another diagnostic mark. One of us is disposed to attach some importance for diagnostic purposes in fevers hitherto little studied to 
the shape assumed by the pigment in the leukocytes, which seems characteristic and uniform for at all events some varieties.

The clinical observations recorded in this paper were made exclusively by Dr. Marshall, who practises medicine at Rio Tinto, in the province of Huelva, in the south of Spain. At certain seasons malarial fever is very prevalent over the whole of that district, and in some parts, which are otherwise healthy, constitutes the chief cause of sickness. Statistics that have been collected show that amongst the working population, although always very prevalent, it has been much more so in those years in which the soil was disturbed by the construction of houses, dams, \&c., and at one time, when medical attendance was much less available than it is now, these fevers were a common cause of death. There are traditions of certain years during which they amounted almost to a plague. Of late years the cases have been less severe and less numerous.

Simultaneously with examinations of the fresh blood by Dr. Marshall, stained specimens from the same cases were studied in London by Dr. Thin, and latterly Dr. Marshall combined the study of the fresh with stained dried preparations. It is interesting to note, as showing the diagnostic value of the microscope in this disease, that at the earlier stage of their studies, important diagnostic points could be determined by the examination of dried and stained specimens in London, and it was found possible, by observing the temperature charts, to select the special cover-glasses which were required for the demonstration of a special stage of the development of the parasite. This was rendered practicable by the time at which each specimen of blood was taken being marked on the chart with a number, the cover-glasses sent bearing the corresponding numbers. After a year spent in general preliminary study of a considerable number of cases, a series were eventually selected for close continuous observation, and from these selected cases a certain 
number have been further chosen for description in this paper as illustrative of the several types observed.

The division of malarial fevers into regular and irregular, which has been made by some authors, is applicable to the fevers which are observed in these districts. The regular fevers are those in which the clinical history and temperature charts show a striking regularity in the time and occurrence of the paroxysm, in the length of its intermission, and the degree of fever, and in this class we include fevers which are recognised even by the people themselves as quartan, tertian, and quotidian fevers. The individual paroxysms in each of these fevers resemble one another so closely as to be indistinguishable one from the other, the points of difference being the length of the intermission between the attacks. Each paroxysm has the well-known cold, hot, and sweating stages, and in any given case these stages may be separately well marked, or the relative severity of the stages, one to the other, may be liable to great variations. The attack is ushered in by pains in the back, neck, and by headache, followed by violent shivering, the thermometer showing, even during the cold stage, a high temperature-104 $\mathrm{F}$. or more. After a cold stage, which lasts not more than an hour and a half, and usually in a much shorter time, the patient begins to feel very hot, has severe headache, and bursts out into profuse perspiration. Then the temperature begins to fall until the patient is free from fever, and is relieved of his painful sensations, although he is weakened by the attack. The entire paroxysm will last from six to twenty-four hours, or longer in a very severe case. The patient suffers most in the cold stage. This stage may be short, and only a slight sensation of cold felt. The hot stage may be long and severe, and the headache intense. The sweating may be so profuse as to saturate the bedclothes.

The paroxysms in the quartan are probably, as a rule, shorter than in the tertian or quotidian.

We have described the chief symptoms of the malarial 
paroxysm as they are observed in Spain, in order to show that they agree essentially with those which characterise fevers of the same type in other parts of the world.

Under the term irregular fevers, we comprise those in which there is no well-marked regularity, either in the time of occurrence of the paroxysm, the length of intermission, or the severity of individual paroxysms. In many cases in which the three stages of the fever are well marked, a considerable irregularity is observed in the temperature chart. Two paroxysms may occur in the same day, or the periods of intermission may be irregular. In some rare cases there is a paroxysm every ten to fifteen days. In others there is no well-marked paroxysm. The cold stage may be absent or be very slight, and the hot stage may or may not be noted by the patient. Sweating may be profuse, or slight, or absent, and one paroxysm may pass into another without any intermission. In these cases the symptoms are usually headache, aching in the bones, and general depression, the patient feeling ill and incapable of doing anything. The appetite is generally poor, and there is a more or less marked cachexia. Under this class come some of the very acute summer fevers, in which there may be no normal temperature registered for a few days, and in which the patient is quite prostrate. In this case there is generally intense headache, and aching in all the body, and the anæmia and the weakness consequent on the attack are very great. In many cases there is in addition to high fever very acute vomiting and jaundice, resembling a severe bilious attack, or there may be choleraic symptoms, which are apt to be misleading regarding the nature of the disease.

Attacks of pernicious fever in which death occurs rapidly whilst the patient is in a state of coma are sometimes found here, but they are rare. 


\section{The Season of Fever.}

In this district fever begins with the hot weather, and occurs with greatest severity during the very hot months, the worst cases being from the middle of July to the middle of September. The first cases of fever show themselves in May, and the malarial season practically ends in the first weeks of October, when the hot weather is over. This refers to the time when the disease may be contracted, fevers rarely beginning during the winter and early spring in people who have not lived for a summer in the districts. A few such cases have, however, been noted. Chronic cases are found throughout the year, but can generally be traced to an acute seizure during the hot months. The length of the fever season depends on the atmospheric conditions, great heat after rains being looked upon as very favourable to the production of fever. A cold summer or one in which there is much rain without great heat is less favourable to its development. A close moist heat is more favourable to the development of the fever than a dry heat. During the colder months quartan fever is the form generally seen, and also cases of low fever associated with cachexia. A chill may provoke an attack in a person who has already had fever or who has been living in the district.

We shall now describe separately the different types of malarial fevers observed in this province, with illustrative cases.

Quartan fevers are comparatively common in this part of Spain. It has been shown by Golgi that a quartan fever is caused by the existence in the blood of a parasite which requires seventy-two hours for its development, each paroxysm being coincident with a division into spores of one generation of this parasite. In a double quartan there are two generations at different stages undergoing simultaneous development. This is the form of fever that is most prevalent in winter and 
spring, and though very chronic in its course, and requiring very energetic treatment for a long time after it seems cured, owing to its great liability to recur, it is the form which produces the least ill effects in the patient, the two days' intermission between each attack allowing him time to recover a certain amount of strength before the next attack. Frequently the patient continues his work, being absent only on the day of the paroxysm, or he may work up to the very moment in which he is attacked with the cold stage.

Many patients have a good appetite between the attacks, and feel fairly well, the only noticeable symptom being a certain amount of anæmia. But in severe cases, and in double quartans, the patient feels ill during the intermissions.

Quinine in large doses in most cases cuts short the attack, but it is always necessary to continue taking quinine at intervals for a considerable time on account of the great liability to relapse shown by this form of malarial fever. The paroxysms occur with great regularity, and the patient can often tell with precision the hour in which he will be attacked. The following cases are related as a sample of the quartan fevers observed in the south of Spain.

\section{Quartan.}

CASE 1.-Josè Fernandez Rosa, æt. 26, a miner, was admitted to the Hospital on November 15th, 1895. He has lived in this district for fifteen years, and was first attacked with fever two months ago, the type being quartan, and the attacks occurring with great regularity at about $4 \mathrm{p} . \mathrm{m}$. on the corresponding days. The patient is a fairly well-built man, but very anæmic, and with a dirty yellow complexion. The spleen is enlarged and tender.

November 15 th.-Temperature 11 a.m., $99 \cdot 2^{\circ}$ F.; 3 p.m., $99 \cdot 6^{\circ}$. Attack began at 3.30 p.m. with pain in the back and 
legs. 4.20 p.m., temp. $105^{\circ} \mathrm{F}$.; shivering violently. 6 p.m., temp. $102 \cdot 4^{\circ}$; sweating, and more comfortable. 12 midnight, temp. $99 \cdot 6^{\circ}$.

Microscopical appearances seen in stained preparations, -4.30 p.m. Small unpigmented parasites in red corpuscles. 8.30 p.m. Small parasite filling one quarter of red corpuscle with very little pigment. These parasites were of probably twenty-four hours' growth, and would correspond to a period of maturity on the 17th.

16th.-Patient was up and feeling well all day; the temperature, except for slight rise to $99^{\circ} \mathrm{F}$. at 8 p.m., was normal.

Microscopical appearances in stained preparations.11.40 a.m. Small pigmented parasites filling one third of red corpuscles. Very young form free. (Two forms of the parasite, a very young form from the spores of the 15th, and a large form, being advanced growth of those observed on the 15th, were distinguished.) 5.20 p.m. Very young forms adherent to the edge of the red corpuscle.

17th.-Temperature rose after 8 a.m., reaching $101 \cdot 6^{\circ}$ at 8 p.m. The patient, however, felt well all day.

- Microscopical appearances.- 4.30 p.m. Parasite filling one third to one haif of red corpuscle. Parasite filling two thirds of red corpuscle with pigment which is not central. These belonged to the generation which would arrive at maturity on the following day. (The sporulating forms causing the slight fever on the 17 th were not. observed, probably owing to the small number in which they were present.)

18th.-Temperature a 8 a.m., $98 \cdot 8^{\circ}$. One gramme quinine sulphate given in solution at 10 a.m. 12 midday, temperature $99^{\circ}$ F. 3.30 p.m., temperature $100.4^{\circ}$; 5 p.m.. $104 \cdot 6^{\circ}$ F. ; 6.45 p.m., $105 \cdot 6^{\circ}$; 9.30 p.m., $100 \cdot 8^{\circ} \mathrm{F}$. Microscopical appearances.-11.25 a.m. Parasite filling four fifths of red corpuscle with peripheral pigment. 3.15 p.m. Sporulating forms. (These forms correspond to the paroxysm on the 18th, the sporulating forms being 
observed just before the onset of the attack.) 6.45 p.m. Skeleton of parasite with central pigment adherent.

19th.-Temperature rose in the morning, reaching $100.4^{\circ}$ at 8 p.m. One gramme quin. sulph. was given at. 10 a.m.

20th and 21st.-One gramme quin. sulph. given each morning at 10 a.m. There were, for a few days afterwards, short rises of temperature to $99^{\circ} \mathrm{F}$., but the appetite continued good, and the patient was discharged well on November 28th. The bowels were costive throughout, requiring an occasional saline draught to move them.

Previous to entering the hospital he had taken quinine at intervals without affecting the course of the fever. Two weeks previous to his entrance one gramme of quinine was given daily for four days in five-grain doses three times a day, but without effect. The same quantity given in the hospital in one daily dose of one gramme effectually cured the fever. It should be noted that the first dose of quinine was given five hours before an access of fever. According to Golgi's observations, the spores of the fresh generation set free on the 18th would meet. in the blood a sufficient concentration of quinine to destroy their vitality at the stage when the parasite is most susceptible to the action of the drug. On the 17th, the day before the crisis, although the patient felt quite well, the temperature rose at $8 \mathrm{p} . \mathrm{m}$. to $101 \cdot 6^{\circ}$, a fact that would indicate that we had probably here to do with a. case of double quartan of which one generation was very weak.

The microscopical observations bear this out, because on the evening of the 15th there were found a few small parasites in a very early stage which would correspond to a period of maturity on the 17th.

That more of this generation were not observed during the 16th and 17th may be accounted for, as already remarked, by the small number in which they 
were present. It may be presumed that a double quartan was just beginning.

The parasite in this case had the recognised characteristic appearance of the quartan parasite, as described by Golgi. The pigment was large and coarse, the spores were large, mostly eight in number, and converged close to a central mass of pigment, and the corpuscle was scarcely enlarged.

CASE 2. Double Quartan.-Jose Pantiga, æt. 13, a bright, fairly well-nourished, but anæmic boy, states that he has suffered from chronic fever of a quartan type at intervals for three years.

This boy came under observation on two occasions, the first being in April, 1895. On this occasion no record of temperature was taken, but the boy and his mother stated that paroxysms occurred on the 17th and 20th April, and this entirely bore out the microscopical appearances observed each morning in fresh blood.

April 17th, 11.30 a.m.-

Microscopical appearances in fresh blood.-a. Sporulating parasites. In some the spores were separate and congregated round the central mass of pigment. There were from eight to ten in each parasite. $b$. Large sterile forms and débris.

These appearances showed that an attack was imminent, and according to the boy's statement it began at 12 noon, half an hour after the observations were made.

18th, 11.30 a.m.-a. Small amœboid parasites with a few granules of pigment in slow movement, occupying about one sixth and one fifth of the red corpuscle. $b$. Débris. This would correspond to twenty-four hours' growth of the spores observed on the 17th.

19 th, 9.30 a.m.-

Microscopical appearances.-a. Parasite filling two thirds of the red corpuscle, with coarse pigment having a sluggish movement. (A more advanced growth of the parasite observed on the 18th : would reach its full 
development and sporulate on the 20th, causing a paroxysm, and this is what occurred.) 20 grains quin. sulph. was given in the morning of the 20th at 8 a.m., and the boy had a severe attack of fever not long after. The next observation was made in the morning of the $22 \mathrm{nd}$.

22nd.-Microscopical appearances.-a. Large sterile parasite. $b$. Pigment-bearing leucocytes.

23rd.-a. Sterile parasite.

25th.- a. Two parasites occupying one third of red corpuscle, with peripheral pigment granules. $b$. Sterile parasite. c. Pigment-bearing leucocytes.

29th.- a. One parasite observed occupying more than one half of red corpuscle. $b$. Sterile forms and pigmentbearing leucocytes.

May 2nd.-a. One parasite seen in red corpuscle. $b$. Sterile forms. c. Pigment-bearing leucocytes.

3rd.-a. One sporulating parasite observed. b. Pigment-bearing leucocytes.

There was a slight attack of fever on the 30th April ten days after quinine, but there was no real paroxysm experienced till May 16th, twenty-six days after.

It will be observed that 20 grains quin. sulph. given in one dose before the attack on the 20th April, whilst it had the effect of practically preventing any paroxsym till the 16th May, still was not sufficient to entirely destroy the parasites. To a great extent, however, it modified the development of those that remained, only a few parasites being observed in a natural state of development, corresponding more or less to attacks which would have occurred had the parasites been present in greater number. The sterile parasites were more abundant, showing that the quinine, though not sufficient to kill, still prevented their true development into spores.

There was throughout only one generation at a time observed in the blood, corresponding very closely in their development to the history given by the patient.

The boy was not again seen till the 20th May, when he was admitted to the hospital. He stated that he had had 
fever on the 16th, and again on the 18th and 19th May, each attack being associated with severe cold stage, headache, and pains in the limbs.

This, which was the type of a double quartan, corresponded to what was afterwards observed while he was an inmate of the hospital.

May 21st.-Temp. 8 a.m., 98.4 ${ }^{\circ}$ F. Between 8 a.m. and $8 \mathrm{p} . \mathrm{m}$. he had a paroxysm, the temperature rising to $101 \cdot 8^{\circ}$ at 2 p.m.

Microscopical appearances in fresh and in stained preparations.-8.15 a.m. a. Sporulating parasites, eight to ten spores. $b$. Parasites filling one half to two thirds of red corpuscles with coarse peripheral pigment. c. Sterile forms. 2.15 p.m. a. Parasite filling half to two thirds. $b$. Sporulating forms. The spores were in many instances separating from each other.

There were here two generations of parasites, one fully developed, causing the fever of that day, the other, two thirds grown, causing the next day's fever.

22nd.-Paroxysm began after noon; temp. $102 \cdot 8^{\circ}$ at 4 p.m., $98^{\circ}$ at 10 p.m.

Microscopical appearances in fresh and stained preparations. -8.20 a.m. a. Sporulating forms. $b$. Small parasites with a few granules of pigment filling one sixth of red corpuscle. Two were observed filling two thirds, with peripheral pigment. c. Sterile parasite. 10.40 a.m. Sporulating forms. 3.45 p.m. a. Young parasite filling one fifth to one sixth with small quantity of peripheral pigment granules. $b$. Sporulating forms with spores separate.

It will be noticed that on this day also there were found two generations of parasites, the one at the sporulating stage causing the fever on the same day, the small forms representing the twenty-four hours' growth of the spores set free on the 21st. The large parasites in the red corpuscles were probably late members of the brood which sporulated on this day.

23rd.-Temperature subnormal all day. 
Microscopical appearances in fresh and stained preparations. -8.45 p.m. a. Young parasites with a few granules of pigment in the periphery. $b$. Parasites filling one half or more of the red corpuscles, with peripheral pigment. c. Sterile forms. [The younger forms here correspond to the spores set free on the 22nd, the other and older form to those set free on the 21st.]

24th.-Temperature rose again after midday, reaching $102^{\circ}$ at 6 p.m. Ten grains quin. sulph. were given at 2 p.m.

Microscopical appearances.-8.30 a.m. a. Sporulating forms. $b$. Parasite with peripheral pigment filling about one half to two thirds of red corpuscle. c. Sterile forms.

Thus the two generations were again found.

25th.-Attack began after noon, temperature reaching $101^{\circ}$ F. at 4 p.m. Ten grains quin. sulph. were given at 10 a.m., and again at 10 p.m.

Microscopical appearanccs.-9.30 a.m. a. Sporulating forms. $b$. Sterile forms. c. Pigment-bearing leucocytes.

Thus only one generation was found, namely, that causing the fever on the same day, no small parasites of the twenty-fourth attack being seen. Ten grains quin. sulph. were given on the 26 th and at intervals afterwards, the patient also taking arsenic in small doses daily. $\mathrm{He}$ was discharged well on the 1st June, having had no fever after the 25th. He was seen some time afterwards and was well, continuing to take quinine at intervals.

The blood was examined occasionally afterwards and the following appearances noted :

27th.-Two days after last attack.

Microscopical appearances.-a. A few sterile forms. b. Two parasites with a very small quantity of pigment.

28th.- a. Sterile forms. b. Two large pheripherally pigmented parasites occupying nearly the whole of the red corpuscle. c. One small parasite in a red corpuscle with quiescent pigment, probably a dead parasite.

30th.- a. One parasite seen with small quantity of pigment. $b$. One sterile form. 
June 1st.-a. Nothing observed.

11th.-Nothing observed.

Here the clinical history and the microscope showed we had to deal with a case of double quartan. It will be seen that on every occasion in which the blood was examined on the 21 st, 22nd, 23rd, and 24th, two generations of parasites were observed, one being twenty-four hours more advanced than the other.

On the 25th it is interesting to note that only one generation was found, it being at the sporulating stage, and being the parasite which caused the fever on the 25th. No young forms were found of the generation which caused the fever on the 24th. It is therefore extremely probable that the quinine given on the 24th, before the fever, had killed the spores set free on that day without affecting to any great degree the generation of two days' growth which caused the fever on the 25th. It is evident, however, that the quinine, with the ten grains given on the morning of the 25th, had the effect of arresting the growth of some of the parasites which belonged to this generation, as the fever did not rise so high nor was the attack so severe as those previously noted, and the sporulating forms found were fewer in number.

Parasites were observed on the 27th, 28th, and 30th April, showing that the quinine given up to that time had not been sufficient to entirely destroy them.

The presence of large sterile forms throughout was indicative of the fact that numbers of the parasites did not undergo their natural development into sporulating bodies and spores.

The preponderance of sterile forms over the developing parasite noted in the later observations illustrates the effect of the quinine. The fact that each sporulating parasite produced from eight to ten spores, of which, in the later days, the most became sterile bodies, would sufficiently explain their number as compared with forms indicative of progressive life. It must also be noted that 
the parasites observed in April were identical in appearance and development with those found in May.

The following summary of the appearances we have observed shows how completely the life history of the quartan parasite of the south of Spain agrees with that described by Golgi as characteristic of the quartan parasite of the north of Italy.

Quartan Parasite, Hemameba malariat (Grassi and Feletti).

In an early stage, when twenty-four hours old, it occupies about one sixth of the corpuscle, which is normal in size and colour. The pigment granules are coarse, and mostly found in the periphery. The parasite has a slow amoboid movement, which gets less active as the development proceeds. The pigment granules have a slow movement, oscillating, or from side to side. The more mature parasite is generally quiescent, although the pigment granules may have a slightly oscillating motion.

The pigment granules remain mostly peripheral till before sporulation, when they begin to congregate in the centre, this being the indication that sporulation is about to take place. The red corpuscles do not enlarge, and though only a rim of red corpuscle may be seen, it has the normal colour.

Sporulation takes place by the division into eight to ten large spores collected round a central mass of pigment. Each spore in fresh blood generally has a brilliant dot, which is its nucleolus. The pigment in some cases is not entirely central, a few granules being scattered between the spores.

The coarse pigment and paleness of the parasite contrast strongly with the normal colour of its host, and make it easily picked out, distinguishing it in this respect from the tertian parasite. The pigment granules are coarser and fewer in number than those found in the tertian parasite, and have not so active a motion. The 
amœboid action is also less active than that of the tertisn at a corresponding stage of development.

The sterile forms are usually larger than the mature parasite. Their pigment may be quiescent, this being the case if the granules are collected together in urasses. In others the pigment granules are scattered evenly throughout the parasite, and have an extremely active movement which has been well compared to the "swarming" of midges. If forms in which the granules are collected together are watched under the microscope, the granules may sometimes be observed to become separate and evenly distributed through the parasite, and then become extremely active.

Teritan Fever is characterised by the well-marked paroxysm which occurs every alternate day, there being an intermission of one day. The length of the intermission depends on the duration of each paroxysm, and may be more or less than twenty-four hours. In a simple tertian the paroxysm will last about twelve hours or longer, and in severe cases the intermission may not reach twenty-four hours. From one paroxysm to another the interval is forty-eight hours.

As Golgi has shown, this form of fever depends on the presence in the blood of a parasite which requires fortyeight hours for its full development, each attack being coincident with the maturation of one generation of the parasite. In this part of Spain its severity varies according to the season. In the spring and early summer the attacks are milder. They increase in severity as the hot weather goes on, until in August and September they may cause very severe and sometimes pernicious attacks. In a double tertian fever there is a daily paroxysm, and two generations of parasites are found in the blood, which reach their full development on successive days. The clinical history of such a case is identical with that of quotidian fever, and it is interesting to note that most of the cases of quotidian fever observed by Dr. 
Marshall at Rio Tinto, in the summer of 1895, and in which the blood was examined, showed that he had to deal with cases of double tertian, as was clearly shown by the presence of two generations of the parasites in two distinct stages of development.

This form of fever produces a more severe effect on the patient than is the case with the quartan fevers; the intermission being shorter gives less time for recovery between the attacks, and the anæmia produced is greater. Though often chronic in its course, it is less so than is the case in the quartans, and it is much less liable to recur if it is promptly and energetically treated with quinine.

The following examples of tertian fever are related :

CASE 3.-Florence Martinez Romano, æt. 20, a miner, a thin, weakly man, was admitted to the hospital on March 5th, 1895. He stated that he had had attacks of fever occasionally for a year, associated with a severe cold stage, and that for the past month he had had it at intervals, the type being quotidian. The spleen was enlarged and easily felt.

March 6th.-Temperature, 12 noon, $98^{\circ}$ F.; 4 p.m., $104 \cdot 6^{\circ} \mathrm{F}$; 8 p.m., $102 \cdot 6^{\circ} \mathrm{F}$; ; midnight, $98 \cdot 4^{\circ}$.

Microscopical appearances in fresh and stained preparations.-2 p.m. a. Sporulating parasites. b. Parasites filling one half to one third with peripheral pigment. 4 p.m. a. Sporulating bodies. $b$. Parasites filling two thirds of red corpuscle, with peripheral pigment. $c$. Parasite filling one quarter to one third, without pigment.

7th.-Noon, temp., $98 \cdot 4^{\circ}$ F.; 4 p.m., $104^{\circ}$ F.; 8 p.m., $100^{\circ}$ F. ; midnight, $98 \cdot 4^{\circ} \mathrm{F}$.

Microscopical appearances.-3 p.m. a. Sporulating bodies and spores. $b$. Young parasites, some unpigmented and some just beginning to be pigmented.

8th.-Temperature, noon, $98 \cdot 6^{\circ}$ F. ; 4 p.m., $105 \cdot 2^{\circ}$ F.; 8 p.m., $99^{\circ}$ F.; midnight, $99^{\circ} \mathrm{F}$.

Microscopical appearances.-10 a.m. a. Young para- 
sites in red corpuscles, some double. $b$. Parasites filling two thirds of red corpuscle with peripheral pigment. Quinine sulphate, gr. 20, given at noon. 2.45 p.m. $a$. Sporulating forms. $b$. Small parasite with faint peripheral pigment. c. One crescent observed.

9th.-Temperature, noon, $98 \cdot 6^{\circ}$ F.; 4 p.m., $104^{\circ}$ F.; 8 p.m., $100^{\circ}$ F.; midnight, $97 \cdot 8^{\circ}$ F. Quinine sulphate, gr. 20 , given at noon.

Microscopical appearances in fresh blood and stained preparations. -9.45 a.m. a. Parasites filling two thirds of red corpuscle. $b$. One crescent. c. Many (sterile) free bodies with scattered pigment. 2 p.m. a. Red corpuscles with small spores. $b$. Sporulating bodies with separating spores. c. Young parasites and sterile forms. The temperature fell at night, and did not rise again, and he was discharged well on the 24th.

Microscopical appearances.-11th.-a. One crescent. $b$. Pigment-bearing leucocytes.

12th.-Four crescents.

22nd.-a. Pigment-bearing leucocytes. $b$. Leucocyte containing a pigmented body resembling crescent. Nothing was observed between the 12th and 22nd, although the blood was examined several times.

The clinical history of this case was that of a quotidian fever, but the microscopic appearances showed it to be really a case of double tertian, two generations of parasites being found in the blood in each examination.

On the 6th, 7th, and 8th, a little time before each attack, there were found the sporulating forms causing the attack, and also the parasites in an earlier stage of development, which would cause the fever of the following day.

On the 8th, 20 grs. of quinine were given four hours before the attack. This dose ought to have killed the spores produced that day, and that they did so is borne out by the appearances noted on the 9 th, when there was only observed one generation, namely that causing the attack. There were also seen sterile forms, showing the 
action of quinine in preventing the natural development of the parasite.

The quinine given on the 9 th destroyed the spores produced that day and cured the tertian fever, as no further rise of temperature was recorded and no parasites were afterwards observed in the blood, except the crescent form which is not connected with tertian fever, and which unfortunately is very resistent to the action of quinine.

Patient was readmitted on May 7th. He stated that he had fever the day after leaving hospital and for nine consecutive days. After this he was without fever till the 5th, when he had an attack, and again on the sixth. The spleen is enlarged and the liver large and tender.

May 7th.-Temperature, noon, $100 \cdot 4^{\circ}$ F.; 4 p.m., $104 \cdot 6^{\circ}$ F.; 8 p.m., $101 \cdot 6^{\circ}$ F.; midnight $97 \cdot 6^{\circ}$ F. Attack ended with profuse sweating.

Microscopical appearances in stained preparations.11.30 a.m. $a$. Parasites filling one third to one half of red corpuscle with or without pigment. $b$. Parasites almost filling red corpuscles with pigment beginning to group, indicative of beginning segmentation. c. One crescent. 1.45 p.m. a. One sporulating form with central pigment and twelve spores. $b$. Parasite filling one third to one half of red corpuscle. 8.30. p.m. Parasites filling one third to one half or more, with peripheral pigment.

8th.-Temperature 8 a.m., $99 \cdot 6^{\circ} \mathrm{F}$.; 12 noon, $105.6^{\circ} \mathrm{F}$. ; 4 p.m. $102^{\circ}$ F.; 8 p.m. and midnight, $99 \cdot 6^{\circ}$ F. Attack ended as on 7 th with profuse sweating. Quinine sulphate 10 grs. given in the evening.

Microscopical appearances.-8.45 a.m. a. Very young parasites with or without pigment. $b$. Mature parasites with pigment combining to form blocks. 12 a.m. Halfdeveloped parasite.

From the 9 th to the 14th there were daily rises of temperature to $100^{\circ} \mathrm{F}$. ; on the 10 th and 11 th it did not fall lower than $99^{\circ} \mathrm{F}$. There was severe cough and slight congestion at the base of the right lung, with inflamed and tender liver. Quinine sulphate 25 grs. 
was given on the 9th in two doses, and after then 10 grs. daily. Patient was discharged well on the 17th, and when last seen two months later was well. The blood was examined on two occasions on the 10th May and nothing observed.

It will be seen that again, as was the case in March, we have clinically a quotidian fever due to a double generation of the tertian parasite. On each occasion in which the blood was examined on the 7th and 8th two different generations of parasites were observed. The history of the case is that of typical double tertian, the symptoms of which, looked at from a clinical point of view only, might legitimately be termed those of a quotidian fever. Microscopical examination, however, showed the simultaneous presence of the tertian parasite in two stages of development, the motile stage observed on a given day indicating the presence of parasites half developed and progressing to the sporulating stage, which would be coincident with a fresh attack of fever on the following day. It must be noted that coincidently with the presence of tertian parasites were found examples of the crescent form of malarial parasite. The exact clinical import of this form of parasite in the present case it is difficult to appreciate as the patient was not sufficiently long under observation, such clinical manifestations of disease as it was capable of producing being masked by the more pronounced symptoms due to the active tertian amœba. The association of the crescent form with the tertian parasite appears to be one of the commonest forms of mixed infection, and affords examples which can be used in support of the theory that one form of the parasite may be transmuted into another. Grassi and Feletti report three cases in which crescents only were found in the blood after a period during which the tertian parasite only had been found. That cases of this kind are, however, due to mixed infection, in which for a time the presence of one parasite is in such small numbers as to be masked by the prevailing form, has, it 
seems to us, been clearly shown both by the observations of pure cases (single infection) and by the results of the artificial production of malarial fever by the injection of malarial blood.

We now give a case in which a typical double tertian was produced by two alternate generations of the tertian parasite uncomplicated by any other form, the case in this respect being typical of many others observed by Dr. Marshall in Spain during the spring and summer of 1895 .

CASE 4.-Manuel S. Sanchez, æt. 26, a strong young miner, has lived for six years in the district, and had fever for the first time a month ago. He took quinine and was then free from it for twenty-six days.

He states that he had attacks on June 17th, 18th, 19th, and 20th. Cold stage is severe, attack generally occuring at midday. There is an herpetic eruption on the lips. He states that the fever has been always quotidian.

June 20th.-When seen at 6 p.m. he was sweating, the attack passing off.

Microscopical appearances. -8 p.m. Parasites filling more than two thirds, with peripheral pigment.

21st.-8 a.m., $96 \cdot 7^{\circ}$ F.; 12 a.m., $104 \cdot 5^{\circ}$ F. ; 4 p.m., $99 \cdot 6^{\circ}$ F. ; 8 p.m., $100 \cdot 6^{\circ}$ F. ; midnight $98^{\circ}$ F. Cold stage began at 11 a.m. and was very severe.

Microscopical appearances in fresh and stained preparations. -8 a.m. a. Large parasites with scattered pigment. $b$. Large sterile forms. $c$. Forms filling one third to one half or more, pigmented. 10.30 a.m. $a$. Sporulating parasite. $b$. Parasite filling one half to one third, with pigment. c. Large sterile parasite. $d$. Segmenting form ; several grains of pigment moving slightly. 4 p.m. a. Parasites filling one quarter to two thirds of red corpuscle. b. Small parasite in red corpuscle, spore size and unpigmented. $d$. Sterile forms. At 8 p.m. Quin. Sulph. 10 grs. given. The generation set free in to-day's attack had already attacked the red 
corpuscles, and would, if not destroyed, have produced fever on the 23rd.

22nd, 12 noon.-Temp. $98 \cdot 2^{\circ}$. Cold stage less severe than last; began at 4 p.m. Temp. 4 p.m., $99 \cdot 6^{\circ}$ F.; 6 p.m., $104 \cdot 4^{\circ}$ F. ; 8 p.m., $101^{\circ}$ F.; 12 midnight, $97 \cdot 6^{\circ} \mathrm{F}$. The attack was here delayed four hours by the quinine given. Quinine sulphate 10 grs. given morning and evening.

Microscopical appearances. -8 p.m. a. Parasites ono third to one half of red corpuscle. $b$. Parasites filling one half to whole, with scattered pigment. c. Large sterile forms-some in red corpuscles which were much enlarged and discoloured, being represented by very faint outlines. 4.20 p.m.-a. Parasites filling one fourth. $b$. Sporulating forms. c. Sterile forms. d. One parasite half filling red corpuscle, pigmented. e. Large parasite with scattered pigment, with outline of decolourised red corpuscle.

23rd.-No fever. Quinine sulphate 10 grs. given morning and evening.

Microscopical appearances.-8.30 p.m. a. Parasite observed filling one quarter of red corpuscle.

24th.—9 a.m. Parasite observed filling one quarter. Quinine sulphate 10 grs. morning and evening. Discharged well on the 27th. On the 24th and 26th only pigment-bearing leucocytes and free pigment seen in the fresh blood.

In this case it will be seen that synchronously with a daily crisis sporulating parasites were present in the blood, accompanied by half-developed parasites which, if their growth went on unhindered, were ready to produce another paroxysm the following day. After the administration of three 10-grain doses of quinine the fever was arrested, and after that time undeveloped and sterile parasites were observed. A very slight rise in temperature $\left(99.5^{\circ}\right)$ on the 24th at midnight, was probably due a very few surviving parasites delayed in their development by the continued administration of quinine. When dis-

vol. LXXIX. 
missed from hospital on 26th he was directed to continue to take quinine for some time, and the cure was complete.

The following case is quoted to illustrate how a certain number of parasites may be present in the blood without invariably producing a febrile attack.

Case 5.-Emenejelva Ameijeiras, æt. 39, has had fever on and off for a year, generally tertian. The cold stage is severe, and the hot stage is accompanied with severe headache. Says he has had fever for four days, but not at regular hours.

July 11th, 11.30 a.m.-Feels hot, but not very ill. Temp. 12 noon, $101 \cdot 8^{\circ}$ F.; 4 p.m., $101 \cdot 2^{\circ}$ F. ; 8 p.m. $101 \cdot 2^{\circ}$ F. ; midnight, $98^{\circ} \mathrm{F}$. Profuse sweating at 8 p.m.

Microscopical appearances.-11.30. a.m. a. parasite filling one third of red corpuscle, with scattered pigment. $b$. One with slight peripheral pigment and numerous oval and irregularly shaped patches in red corpuscle stained blue (methylene blue staining). c. Sporulating body; spores separate. 4 p.m. a. Small number of parasites half developed. $b$. Pigment-bearing leucocytes.

12th.-Purge of Magnes. Sulph. No ferer all day.

Microscopical appearances. -9.30 a.m. a. (In fresh blood), small parasites with very fine pigment. $b$. Segmented forms. c. Sterile form. 4.30 p.m., several halfsized parasites.

13th.-Fever came on at 9 a.m. with severe shivering, Temperature 8 a.m., $98 \cdot 6^{\circ}$ F.; $11.30,104^{\circ}$ F.; 4 p.m., $99.6^{\circ}$ F.; 8 p.m., $99^{\circ}$ F. Quinine sulphate, 15 grs., at 8 p.m., and on 14th at 8 a.m.

Microscopical appearances.-9 a.m. a. Sporulating bodies, one with sixteen spores. $b$. Young parasite. $c$. Pigment-bearing leucocytes. Under administration of quinine 15 grs. daily the fever was arrested.

It will be observed that although sporulating forms were found on the 12th, no febrile paroxysm occurred on that day. The generation due for that day had already been olicerved to be scanty on the 11th, and the man had 
been freely purged on the morning of that day, two facts which must be borne in mind in connection with the absence of a paroxysm.

\section{The Teritia Parasime, Hamameba Vivax (Grassi and Feletti).}

This parasite, which undergoes development in twentyfour hours, is found in the early stage as a small, round, pale body adhering to the red corpuscle. Later it is found within the red corpuscle, which becomes progressively enlarged and paler with the growth of the parasite, till in the mature forms only a small rim of corpuscle is found, almost entirely deprived of hæmoglobin. The young parasite has a most active amcboid movement in the red corpuscle, throwing out arms in all directions and assuming the most fantastic shapes. As it enlarges it becomes less active, till we come to the mature sporulating body which is quite quiescent.

Pigment early shows itself in very fine granules, contrasting in this respect strongly with the coarser granules of the quartan. This pigment has a most active, swarming motion, and retains this property, though to a less extent, up to maturity, when it becomes still and congregates in the centre preparatory to sporulation, which takes place by the division into twelve to sixteen spores, that are smaller than those of the quartan.

The features distinguishing this parasite from the quartan are as follows :

The amcoboid movement is more active than in the quartan, in which it is comparatively slow.

The pigment is finer at corresponding stages, and has a very lively movement.

The spores are small in size, but more numerous than those of the quartan.

The red corpuscle, which in the quartan is little, if at all, altered in size and colour, in this case becomes very 
early enlarged and pale, and by not offering a sufficient contrast, renders the parasite more difficult to find in fresh preparations.

The sterile forms resemble those found in the quartan.

\section{IRREGULAR FEVERS (INCLUDING TRUE QUOTIDIAN FEVERS).}

We shall now give a few examples of the fevers which in the present condition of our knowledge may be provisionally and conveniently termed irregular fevers. They are frequently chronic, and are often obstinate, as the parasites are not so easily destroyed by quinine as in the tertians and quartans. Sporulation takes place in the internal organs, the blood from the finger seldom showing sporulating forms, the parasites which are found in it being mostly in an early stage of development, and often very few in number. It is in a certain proportion of these forms of fever that the crescent body is found, and in which extensive changes are produced in the spleen and liver by the development of the parasites in the tissues. Severe forms of fever-the types often termed " remittent,"-and forms of malarial cachexia without febrile attacks, are both due to the parasites of this class. These irregular fevers manifest themselves in the south of Spain in summer and autumn, but being exceedingly chronic and obstinate are found in patients at all seasons of the year. The cases found in winter and spring are those which had begun at the end of the previous summer, and have remained uncured. They correspond in this and in many other respects to the summer-autumn fevers of the Italian physicians. The following cases are examples of these irregular fevers as observed in the south of Spain.

Case 6.-Francisco Nunez Rodriguez, æt. 42, a miner, admitted December 5th, 1894. 
The patient, a weakly, thin man, with a very dirty sallow complexion, has suffered more or less from fever since August 27th. 'The type has varied, and quinine for a time cut it short, but it soon returned. For the last few days he has had daily attacks beginning in the afternoon. Three days before admission he took a purge and 6 grs. quinine daily for the three days. Lungs healthy.

December 5th.-10 a.m., temp. 98.4 $4^{\circ}$ F.; 12 noon, temp. $98.4^{\circ}$ F. 2.30 , pains in bones, and tenderness over spleen. 4 p.m., temp. $100 \cdot 1^{\circ} \mathrm{F}$; ; has had slight sensation of cold. 8 p.m., temp. $102 \cdot 8^{\circ} \mathrm{F}$.; feels warm, less pain. Slight sickness during night, then sweating, and relief from all pain.

Microscopical appearances.-10 a.m. Parasite occupying one third with fine pigment points. Noon. a. Unformed masses with pigment. $b$. Small parasites filling one third to one sixth of red corpuscle. 4.10 p.m. a. Unformed masses free in the blood, containing small rounded clumps with pigment granules attached. $b$. Small free clump with pigment attached, apparently débris of a sporulating parasite.

6th.-9.30 a.m. Comfortable; temp. $99 \cdot 8^{\circ} \mathrm{F}$.; 12 noon, temp. $99 \cdot 2^{\circ}$ F.; 4 and 5 p.m., temp. $100 \cdot 6^{\circ}$ F. Slight shivering and headache in afternoon. 8 p.m., sweaving, temp. $100^{\circ} \mathrm{F}$; midnight, temp. $98 \cdot 4^{\circ} \mathrm{F}$.

Microscopical appearances in stained preparations.9 a.m. a. Parasite filling one half, with central commashaped pigment. $b$. Blue-stained body, filling nearly one half of red corpuscle. 12 a.m. Small spherical body with central pigment.

7th.-Temperature normal and subnormal till afternoon. 4 p.m. Temp., $988^{\circ}$ F.; no cold or pain. 8 p.m. $100 \cdot 6^{\circ} \mathrm{F}$. Midnight the same. Sweating at night. 9 a.m. One gramme quinine sulphate in solution given.

Microscopical appearances.-9.30 a.m. Spherical agglomeration of pigment in fine granules in centre of red corpuscle, indicative of crescent formation. 4 p.m. $a$. 
Ruptured red corpuscles. $\quad b$. Crescent. $c$. Sinall round parasite.

8th.-9 a.m. One gramme quinine sulphate given. Temp. 4 a.m., $99^{\circ}$ F.; 8 a.m., $99 \cdot 4^{\circ}$ F.; noon, $99^{\circ}$ F.; 4 p.m., $101.4^{\circ}$ F. ; 8 p.m., $99 \cdot 4^{\circ}$ F. ; midnight, $98 \cdot 8^{\circ}$ F. Slight attack preceded by headache and sensation of cold. Short hot stage which ended by sweating.

Microscopical appearances.-10 a.m. a. Crescent. b. Small round parasite. c. Parasite filling one fifth of red corpuscle with central pigment. 4.30 p.m. a. Crescent. b. Small parasite.

9th.-Temperature subnormal and normal. Headache and deafness. Sweating at night slight.

10th.-Condition the same as on the 9th. Temperature rose slightly in the afternoon. 9.30 a.m. a. Small spherical body in red corpuscle. $b$. Crescent. c. Small parasite.

13th.-Temperature normal.

Microscopical appearances.-9.45 a.m. a. Crescent. b. Red corpuscle containing spherical pigment in centre (outline of parasite not well defined). c. Small parasite.

14th.-Temperature normal.

Microscopical appearances.-9.45. a. Small, free, rounded clumps with pigment granules attached. $b$. Crescents and small parasites.

17th.-Discharged. Feeling well when seen some months after.

There is to be noted in this case that the parasites found in the blood from the finger were small, and in an early stage of development; that some parasites were found with the spherical central mass of pigment, which is distinctly indicative of crescent formation; that crescents were found free in the blood; then after rest in the hospital and administration of two doses of 15 grs. quinine, the temperature became normal, and the fever crises ceased, but the blood continued to show crescent forms. The patient was seen occasionally for four months afterwards, and appeared to be well. No 
more fever attacks occurred, but there was no opportunity of examining the blood.

A point of interest in this case, as regards the microscopical appearances, is the fact that the parasites in the blood from the finger were found either in a condition of small bodies, or bodies filling half the red corpuscles containing pigment, or crescents.

The clinical symptoms and the temperature chart are those of a quotidian type of fever, and the forms of the young parasites found would not be inconsistent with those described as characteristic of the quotidian parasite by the Roman school. It would be quite in accordance with the teaching of that school to find crescents developing from the quotidian parasite. On the other hand, there is nothing in this case that is incompatible with the theory of Grassi and Feletti, that in instances like these we have to do with the crescent parasite as a separate species-genus Laverania, species Laverania malariæ, of these authorsand the young forms would be regarded throughout as early forms of the crescent body. It will be observed that no sporulating form was found in the blood from the finger.

CASE 7.-Juan Garrida Martin, æt. 18, admitted December 20th, 1894. States that he has been suffering at intervals for four months from fever, and that on the 18th and on the 19th he had a paroxysm.

December 20th.-Temperature 1 p.m. $98 \cdot 6^{\circ}$ F.; 3 and 9 p.m., $98^{\circ}$ F. ; midnight, $101 \cdot 4^{\circ}$ F. Mild paroxysm.

Microscopical appearances in stained preparations.3 p.m. Number of red corpuscles with round and horseshoe-shaped pigment in a small clear space in the corpuscle. 4.30 p.m.- $a$. Parasite filling one half of the red corpuscle with central pigment. $b$. Parasite nearly filling red corpuscle with fine peripheral pigment. $c$. Parasite filling one quarter of the red corpuscle with nine minute hæmatoxylin-stained peripheral points in its circumference.

21st.-Temperature 9 a.m., $96 \cdot 6^{\circ}$ F.; 4 p.m., $99^{\circ}$ F.; 
8 p.m., $99^{\circ}$ F.; midnight, $104 \cdot 6^{\circ}$ F. Rather severe paroxysm.

Microscopical appearances.-10 a.m. a. Spherical body with central point of pigment nearly filling red corpuscle. $b$. Round body with peripheral pigment filling two thirds of the red corpuscle. 2 p.m. a. Free oval body, smaller than a red corpuscle, with very fine pigment. 4 p.m. Minute spherical bodies in red corpuscles with central point.

22nd.-4 a.m. Temp. $100 \cdot 6^{\circ}$ F. ; 8 a.m. to midnight, temperature $98^{\circ} \mathrm{F}$. No paroxysm this day.

Microscopical appearances.-10 a.m. a. Hæmatoxylinstained body filling three quarters of a red corpuscle with two more deeply stained points in the periphery. b. Many minute pigment points in clear spaces in red corpuscles. 2 p.m. a. Two parasites occupying three fifths of a red corpuscle, apparently joined in the centre. $b$. Hæmatoxylin-stained body nearly filling red corpuscle (? dead parasite). 7 p.m. a. Many minute pigment points in clear spaces in red corpuscles. $b$. Great variety of small stained bodies with small hæmatoxylin-stained points. c. Hæmatoxylin-stained body filling two thirds of a large red corpuscle, with scattered pigment. $d$. Not well defined appearance of sporulation. e. Small parasites filling one fifth to one quarter of the red corpuscle. $f$. Small parasites on the periphery of the red corpuscles.

23rd.-Temperature 4 a.m., $102 \cdot 8^{\circ}$ F. ; 10 a.m., $98 \cdot 4^{\circ}$ F. ; normal for the rest of the day. Slight paroxysm.

Microscopical appearances.-10.30 a.m. a. Small parasite. $b$. Parasite filling one fourth to one third of the red corpuscle with central pigment.

The patient here passed out of observation till December 29th, owing to the absence of Dr. Marshall.

On the 24th there was a double paroxysm with a distinct intermission for about an hour.

25th.-Short paroxysm with high temperature-104.6 $6^{\circ}$ 9 a.m.; temp. $98^{\circ} \mathrm{F}$. at 4 p.m. ; $11.30,99 \cdot 6^{\circ} \mathrm{F}$. 
26th.-Temp. $101 \cdot 3^{\circ}$ at 4 a.m.; $99 \cdot 2^{\circ}$ F. at 8 a.m. and 4 p.m.; $98^{\circ}$ F. at midnight.

27th.-Severe paroxysm; temperature reaching $104 \cdot 4^{\circ}$ at midday. Ten grs. quinine given at 9 p.m.

28th.-Temp. $98^{\circ}$ F. at 9 a.m. ; 10 grs. quinine sulph. given. Very severe paroxysm. Temp. $105 \cdot 6^{\circ}$ at 4 p.m.; 10 grs. quinine given in the evening.

29th. - Microscopical appeurances. - A parasite was found filling one third of red corpuscle.

The temperature remained from this time normal, and he was discharged on January 2nd apparently well.

A glance at the Chart in this case, with a consideration of the symptoms, will show that it answers to the description of quotidian fever as given in Marchiafava and Bignami's work. The parasite as we have described it corresponds sufficiently accurately to the quotidian parasite of these authors, the Hæmamoeb a præcox of Grassi and Feletti. It will be observed that although the blood was examined twice, and sometimes three times daily from the 20 th to the $23 \mathrm{rd}$, and again on the $29 \mathrm{th}$, no crescent forms were ever found. Nor can it be said that this was due to the short duration of the fever. According to the man's own statement he had paruxysms two days preceding his admission, and he had a daily paroxysm in the hospital until the 28th of December, so that although there were at least ten days' fever the parasites did not develop into the crescent form. The Roman school would not consider that to be inconsistent with their doctrine, for Marchiafava and Bignami state that there are a considerable number of cases in which the crescents do not appear. On the other hand Grassi and Feletti would explain this case by considering the parasite to be of the pure quotidian variety without any admixture of the crescent species, and that the reason no crescent forms were found was becanse that variety was not present in the blood, the quotidian never being transformed into that species. In this case there is to be noted the irregularity of the crisis, the number of small parasites, 
and the comparative absence of sporulating forms in the blood from the finger, only one being found.

The condition of the pigment in the parasite in this case is characteristic and deserving of notice. In many instances its presence in the red corpuscles was shown by a small colourless sphere which contained in its centre a minute comma or horse-shoe shaped mass of homogeneous-looking pigment without any appearance of sporulation, contrasting in this respect not only with the arrangement of pigment in the quartan and tertian parasite, but also in that of some other cases of irregular fever. One of us has found similar appearances in the blood of cases which have occurred in the West Coast of Africa, and this peculiar arrangement of pigment would seem to have a certain diagnostic bearing.

There are cases of long-continued chronic fever in which the patient feels out of health, but in which the rises of temperature are small and infrequent. The crescent body is not always found in these cases in blood taken from the finger, and it is not always easy to determine from the parasites which are found in the blood to what variety they belong. The following case is given as an example of the difficulty that may exist for a time in establishing the final diagnosis. No crescent forms were found, and only one form resembling a sporulating body with central pigment.

CASE 8.-Josè Sanchez Concession, æt. 24, admitted April 18th, 1895.

Patient states that he has suffered at intervals for a year from malarial fever of tertian type, that the present attack dates from the 11 th, and that paroxysms occurred on the 13th, 15th, and 17th.

$\mathrm{He}$ is weak looking and cachetic. Spleen enlarged. During his stay in the hospital he had no attack. The temperature rose for an hour or two daily to $99^{\circ} \mathrm{F}$. On the 24th and 28 th it rose to $100.2^{\circ} \mathrm{F}$. without a cold stage (see Temperature Chart). On the 27th he com- 
plained of pain in the spleen, for which an ointment of belladonna and hydrarg. was ordered. Discharged on May 7th well and stronger.

No quinine was given or taken for some time before admission. Tonics were given.

Microscopical appearances.-April 18th.-10.30 a.m. $a$. Several parasites filling more than two thirds with peripheral pigment. $b$. One filling more than one half; no pigment. c. Parasite filling one third in enlarged pale red corpuscle. $d$. One segmented body. 3.30 p.m. $a$. Large parasite with scattered pigment. $b$. Small parasite filling one third with pigment. c. Free small parasite with filaments (flagella).

April 19th.-10.30 a.m. a. Small spherical body with peripheral pigment points. $b$. Parasite filling one third, pigmented. c. Large parasite filling red corpuscle, with scattered pigment. $3 \mathrm{p} . \mathrm{m}$. a. Parasite filling one third to one half. $b$. Several parasites with very little pigment.

20th.-8.30 a.m. a. Parasite filling one third to one half or more with very fine, scattered pigment. $b$. Small parasite.

21st.-11 a.m. a. Stained parasite filling one third of red corpuscle. $b$. Irregular shapes one quarter to one third with fine peripheral pigment.

22nd.-9 a.m. Same as above.

24th. - 9.30 a.m. a. Parasite nearly filling red corpuscle with two minute dots of pigment. $b$. Red corpuscle two thirds filled with bluish-stained body (hæmatoxylin); no pigment. c. Three stained small spherical bodies in a red corpuscle touching each other.

26th.-10 a.m. a. Parasite filling from one third to one half ; no pigment.

29th.-3 p.m. Several parasites filling one quarter to one third or more, pigmented.

30th.-8.30. 'Three sterile bodies.

We give another similar case because a crescent body was found with parasites which resemble the early forms of the tertian parasite. 
CaSE 9.-Manuel Trigo Fernandez, æt. 16, admitted June 7th, 1895. States he has had fever for five months, on and off, of a tertian type. He has taken quinine during that time, but none for the last week. He had attacks on the 2nd, 4th, and 6th, with severe pain in the head and slight cold stage, but no stage well marked. Two years ago he had tertian fever for seven months.

June 8th.-Temp., 8 a.m., $98^{\circ}$.F.; 12 noon, $99.8^{\circ}$ F.; 4 p.m., $100 \cdot 4^{\circ}$ F.; 8 p.m., $98 \cdot 2^{\circ}$ F. ; 11 a.m., pains in limbs and head; attack mild.

Microscopical appearances.-7th.-10 a.m. (Stained preparations.) a. Small circlet with more deeply stained, small, rounded body in the ring, on the border of a red corpuscle. $b$. Clear space containing hæmalum-stained body filling two thirds of a red corpuscle. c. Parasite filling one half. $d$. Small parasite filling one fourth, pigmented. e. Large parasite filling two thirds with scattered pigment. 4 p.m. a. Red corpuscle filled with large sterile parasite (scattered pigment). $b$. Crescent. $c$. Red corpuscles with pigmented parasites filling one third to one half. $d$. Sterile forms.

8th.-8 a.m. a. One small parasite in enlarged red corpuscle with slight pigment. $b$. Large red curpuscles with parasites filling one half or more, with peripheral pigment. c. Sterile forms. 3 p.m. a. Small unpigmented parasite in red corpuscle. $b$. Large forms filling three quarters to whole. $c$. Sterile forms.

9th.-Temp. $99 \cdot 6^{\circ}$ F. at 8 a.m.; normal for rest of day.

10th.-Temp. $99 \cdot 4^{\circ}$ till 8 p.m. (thereafter normal till discharge).

Microscopical appearances. -8.45 p.m. One large parasite occupying three quarters of red corpuscle.

The case was very mild. The boy was well nourished and had no enlargement of the spleen. He had slight anæmia. Fifteen grains quinine given on the morning of the 10th. Ten grains of quinine in two doses daily on 11 th and 12th, on which day he was discharged.

It is to be noted that in this case sporulating forms in 
the blood from the finger were not found. The case was not well marked, the temperature was not high, and a crescent form was found, indicating that the patient was the subject of chronic malaria. What relation the presence of the crescent body had to the febrile attacks it is diffcult to say, but it had an undoubted relation to the anæmic condition. Possibly a more exhaustive examination of blood from the finger might have resulted in the discovery of a sporulating form.

Now that it has been definitely established that malarial fever is due to a hæmatozoon, the question as to how it gets introduced into the human body becomes very prominent. Various factors have been long known to be concerned in the production of malaria, particularly certain conjoint conditions of temperature and moisture. These conditions we now recognise must owe their power to the influence which they have on the development of the hæmatozoon, but how the parasite gets introduced into its host is yet unknown. It becomes interesting, therefore, to examine carefully once more all the conditions that are known to favour the development of malaria, with special reference to this problem. For this reason we shall notice some of the conditions that are associated with malarial fever in the south of Spain, our experience being limited to that part of the Province of Huelva in which the observations were made.

\section{SoIL.}

The aspect of the country is that of a series of short hills, with intervening ravines or valleys, in many of which there are streams in winter. These dry up in the summer, leaving pools. There are no marshes. It is about 320 mètres above sea level. The soil is firm, and water does not lie on it long. There is no vegetation on account of sulphur smoke, but about twenty years ago it was largely wooded, and rich in orchards. 
Some of the villages are built on the face of a small hill, with a watercourse at the bottom. The houses inhabited by workmen have, for the most part, earthern floors, or stones or tiles placed on the earth. The streets in the villages are unpaved, or have only stones set into the ground. The houses in the town of Rio Tinto have floors of flat tiles.

The houses in village $A$ have wooden floors on the ground floor. Here the men for the most part sleep on the first floor. In the town they sleep on the ground floor.

In the surrounding villages the houses are one-storied.

It is interesting to observe that here, as in other parts of the world, the malaria is often found to be strictly localised, a very short distance being often sufficient to divide a fever-stricken from a comparatively fever-free situation. Although we have not been able to discover differences between fever-free and fever-producing localities which are near each other, it may be useful to emphasise the fact that these differences exist by relating what has come under our own notice.

\section{Localisation.}

It is well recognised in the district that there are some spots where malaria is always prevalent in the fever season, and others, as for example the town, where it is not found to any great extent. In fact people in the town run little danger of catching the fever, while people living in village $A$, half a mile off, even although they sleep on the first floor, are more liable to be attacked. For example, an Englishman who always had fever when living in village $A$, when he removed to the town was seldom affected. Another man who lived six years in the town without catching fever, went to live at village $A$ and was attacked. To illustrate how narrowly the infection may be localised we may refer to two villages which are distant from one another about a quarter of a mile. $B$ is on a plateau; $\mathrm{C}$ lower down on the other side of a stream, and on the 
side of a hill. $\mathrm{C}$ is notoriously malarial; $\mathrm{B}$ is much more healthy, although the houses are similar in each.

About 300 yards from the village $A$, there are houses on a small elevation with a watershed on each side, inhabited by foreign workmen. Here fever is very common, and three people out of a group of eight have been affected with fever at one time.

Most of the malarial villages are situated on the face of a hill, with a stream, which is more or less dried up in summer, at its foot. In the same village there are streets and even houses where it is more frequently met with than at other places. Sometimes in a house liable to the infection Dr. Marshall has seen four patients suffering simultaneously from fever, and it is not at all uncommon to find unore than one patient down with it at the same time in the same house or same room.

Facts such as these would seem to indicate that the parasite, in the stage in which it gets admission into the circulation, not only hugs the soil, but that it is not readily carried by currents of air from one part to another, and we may reasonably suppose from the facts observed here (as elsewhere), that moist conditions possibly produce some change in the parasite in its extracorporeal life, and that those changes render it more fitted to establish itself in the human body.

Here, as in other countries, change of locality often has an excellent result in curing the fever. For instance, a Spanish servant was ill in one street with fever, and when removed to another house she almost at once lost it. Two Englishmen at Punta Umbria, with a slight form of fever every day, ceased to have attacks from the day they were removed to Huelva Hospital. Such recoveries are too rapid to be entirely dependent on removal from sources of infection, and must be largely attributed to the tonic effect of change.

Disturbance of soil is a well-known cause of activity in the infection by the malarial parasite, and this has been observed in this district. During certain seasons in 
which there had been extensive foundations excavated for dams and houses, and when the houses in certain villages were first built, there was much more fever than generally has occurred in them since. One Englishman was seriously attacked with fever while superintending the making of a tennis-court in front of his own house for which much soil had to be removed.

We can only account for the disturbance of the soil producing fever by presuming that it sets free into the air a number of malaria parasites in a condition ready for beginning their intra-corporeal life, and although the many researches which have been made with the object of detecting the parasite in the soil have not, so far, been conclusive, it is evident that this is a line of research which will not be abandoned until the problem has been solved.

Drinking-water has often been suspected of being the means by which the parasite is admitted into the body. Hitherto this has not only not been proved but there are many facts which render it improbable. The hypothesis is certainly not borne out by what has been observed in the district where our cases were observed.

The drinking-water in this district is pumped out of wells. All the English people are supplied from the same wells, although living in different parts of the district; yet those persons who live in the town are remarkably free from fever, whilst those in village $A$ and those living on the elevation 300 yards from it, before alluded to, are more liable to the infection.

The two villages $\mathrm{B}$ and $\mathrm{C}$, contrasted before, get water indiscriminately from the same two wells, yet one is more seriously affected with fever than the other.

So far there is no sufficient evidence that the parasite gets into the system by the mouth. The experiments of the Roman School of Hygiene are against water containing the infection (Grassi and Feletti); pigeons which were made to swallow dew collected in malarial districts remained immune. Calandruccio and Grassi drinking 
from 30 to 50 grammes of dew also remained immune, as did Calandruccio after swallowing considerable quantities of mud which might well have been considered to contain the malarial poison. Nay, even malarial blood may be swallowed both by human beings and pigeons without causing malaria. (Pigeons and some other birds are affected in malarial districts with a hæmatozonn similar to the hæmatozoon of malaria in man).

Laveran, considering that mosquitoes are found in malarial districts, and that draining the soil, which prevents fever, also destroys mosquitoes, suggests the possibility of these insects acting the part of an intermediary host for the malarial parasite, as they do for the filaria. Grassi and Feletti object to this hypothesis from the fact that birds which become infected with hæmatozoa in malarial districts are not attacked by mosquitoes. A pigeon, in which the blood is ascertained to be quite healthy, when suspended at a certain distance from the ground over a malarial soil, becomes the subject of a hæmatozoon which if not identical with that of the hæmatozoon which attacks man, is allied to it, and mosquitoes and insects can hardly, in this case, be held responsible for the infection. Further, Calandruccio, who has investigated the subject, states that the malarial parasite dies in the intestine of the mosquito without undergoing any further development, the same fact being verified for the leech. It is further well known that, as Grassi and Feletti remark, many places are full of mosquitoes without having malaria. Malaria exists in Sicily in places where there are no mosquitoes that bite. (Oral communication by Prof. Feletti.) It was stated by Dr. Manson, at the annual meeting of the British Medical Association in 1895, that Surgeon-Major Ross, working in India, had found that the parasites are able to resist destruction in the stomach of the mosquito, and considers that a development in this insect as an intermediate host is evidenced by the fact that a flagellate form is more frequently found in blood which has been, in the mosquito than vol. LXXIX. 
in blood from the finger, and that the crescent forms all become spheres. A full account of the paper in which this statement is included has not yet been published, but so far as these two statements go they are not convincing. The right appreciation of phenomena of this kind requires the special knowledge of the lower forms of life which is possessed by zoologists. On such a point, therefore, the association of Professor Grassi in the memoir bearing his name with that of Professor Feletti deserves much consideration.

These authors remark that the assumption of the rounded form may indicate approaching death in many protozoa (Mastigofori, Amœba, \&c.), and that at the same time that the crescents throw out flagella they throw out round hyaline bodies, three, four, or more, of different sizes (the cytoplasma of the parasite), similar bodies being thrown out by many protozoa. In comparison with the setting free of flagella by the flagellata, they state that in the case of a crescent body the separation of the flagellum follows too soon on its production. They quote instances in spermatozoa which undergo similar changes and movements in the death agony.

From the facts which they adduce they conclude that certain forms of life, and certain organised elements when placed in abnormal media, react by unusual and irregular movements, which usually precede death. The crescent forms in blood removed from the circulation thus find themselves, from the blood coagulating, in an abnormal medium, and it is not to be wondered at that they change into flagellated bodies before dying.

These are points on which we do not consider that our opinion is entitled to weight. We do not lay claim to special zoological knowledge, and we have not applied ourselves to the study of this particular question. The subject is, however, so important, that our paper would be incomplete without a reference to it. The instance of the man cited by Surgeon-Major Ross, who, eleven days after swallowing a bottle of water containing malarial 
mosquitoes, developed malarial disease, is on the other hand, of importance. As a solitary instance it cannot claim great weight, but if confirmed by similar examples it would prove to be a fact of much value, as indicating that in addition to the ordinary exogenous development of the malarial parasite, which undoubtedly exists, the hæmatozoon could also be conveyed through water by the mosquito.

The mosquito theory does not find much support in the district we are considering. Mosquitoes are indeed found, but not in numbers sufficient to be unpleasant, mosquito nets not being necessary. Flies are more common, and many of them bite.

The sulphur smoke and consequent want of vegetation account for the comparative absence of insect life, although they do not seem to have any specially injurious effect on the extra-corporeal phase of life of the parasite of malaria.

(For report of the discussion on this paper, see ' Proceedings of the Royal Medical and Chirurgical Society,' Third Series, vol. viii, p. 125.) 


\section{DESCRIPTION OF PLATE I,}

The Parasite of Malaria (Robert J. Marshall, M.B., C.M., and George Thin, M.D.).

Fias. 1 to 14 represent the quartan parasite from its earliest stage after attacking the corpuscle to its full development by the production of a new generation of spores.

FIGs. 15 and 16 represent free pigmented bodies in the blood, which we consider as sterile forms of the quartan parasite.

FiG. 17 represents small pigmented bodies with pigment granules found free in the blood in quartan fever.

(These drawings were made by Dr. Marshall from preparations of fresh blood.)

Fifs. 18 to 29 represent the progressive development of the tertian parasite from its first attacking the red corpuscle to its complete development into free separating spores.

FIGs. 30 and 31 represent free pigmented bodies of the tertian parasite, which we consider to be sterile forms.

FIG. 32 represents pigmented bodies relating to the tertian parasite of various sizes found free in the blood. In one of these a long flagellated process is seen with swellings.

(These preparations were all drawn from the fresh blood, except Figure 28, which was drawn from a dried and stained preparation from the same patient as the others.)

FIGs. 33 to 48 represent the appearances presented by the parasite in a case of irregular fever. Many crescents were found in the blood. No sporulating forms were found. Transition forms from the small colourless amœba to appearances indicative of the crescent form are already evident in Figures 38 and 39.

(They were all drawn from the fresh blood, except Figure 48, which was drawn from a stained preparation to fill up a gap in the forms observed in the fresh blood. This is one of the cases which have not been described in the text.)

FIGs. 49 to 63 represent the parasite as seen in a case of irregular fever in which no crescents were observed.

FiGs. 49 to 53 represent appearances seen in blood preserved and examined in Pacini's fluid, the parasites being represented by a colourless space in which the dark pigment is shown very distinctly.

FIG. 54 represents a small pigmented mass seen in blood from the same case preserved and examined in Pacini's fluid.

FIGS. 55 to 63 represent parasites from the same case in dried and stained blood preparations, the staining being effected by various hæmatoxylin methods.

FIGs. 56 to 60 show distinctly stained nuclear or nucleoliform elements.

FIG. 61 shows stained thickened points in the periphery of the parasite.

FIG. 62 shows a parasite with very fine pigment scattered through it.

FIG. 63 represents a sporulating form. This was the only sporulating form observed in a very large number of preparations which were very carefully examined.

FIGs. 64 and 65 represent circular bodies with stained points in blood from the same patient. They are drawn on a much more highly magnified scale than the other drawings in order to bring out very clearly the stained points. Their actual size was not a third of a red corpuscle. Similar small bodies with stained points have been described by other authors. They are quite distinct in appearance as well as in size from the ordinary sporulating forms.

It will be observed that in this case the forms of the parasite seen nearly all represented very early stages, Figures 62 and 63 being quite exceptional, showing that only the very young forms were to be found in the peripheral circulation. 


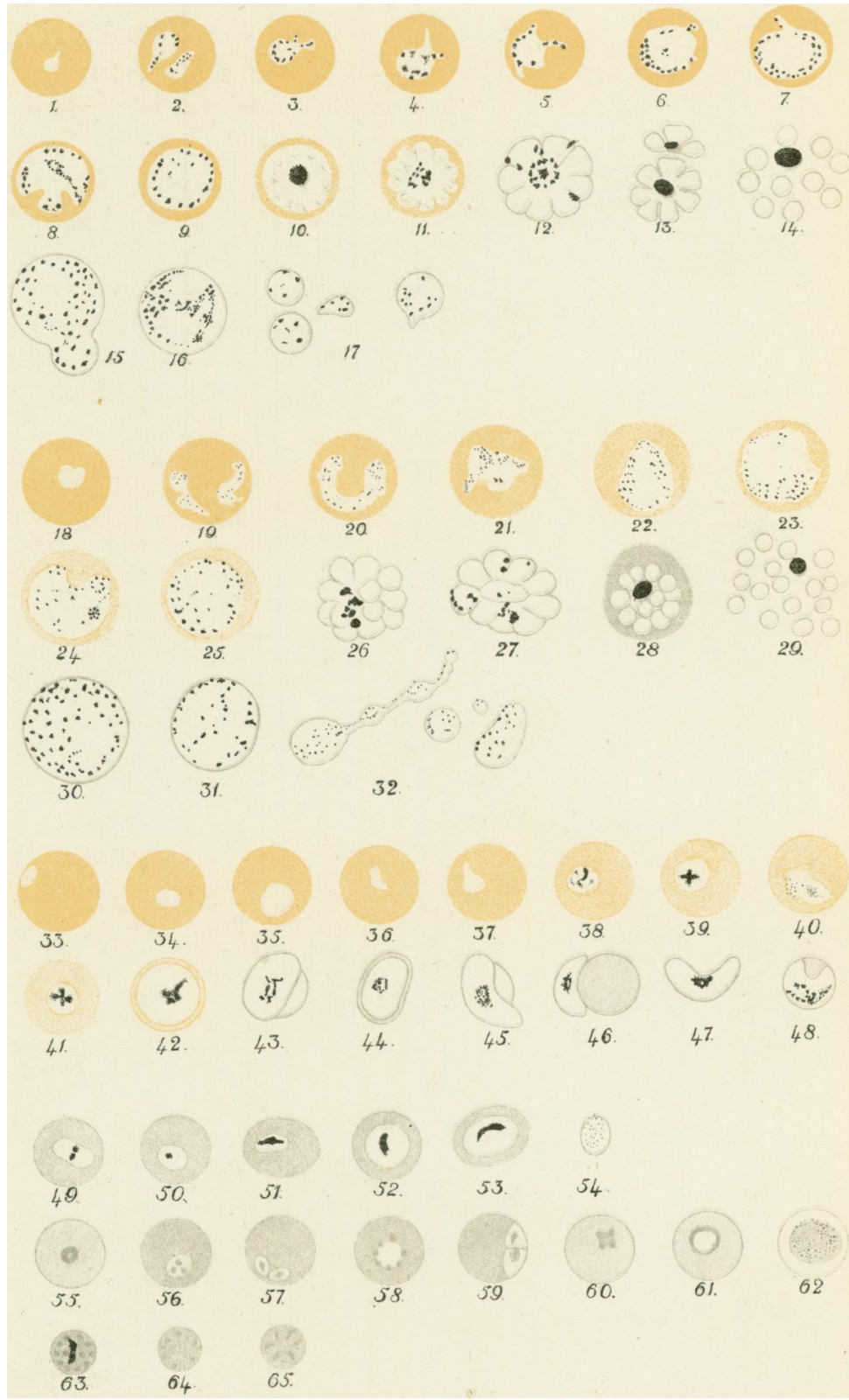


CASE 1.-Josè Fernandez Rosa.

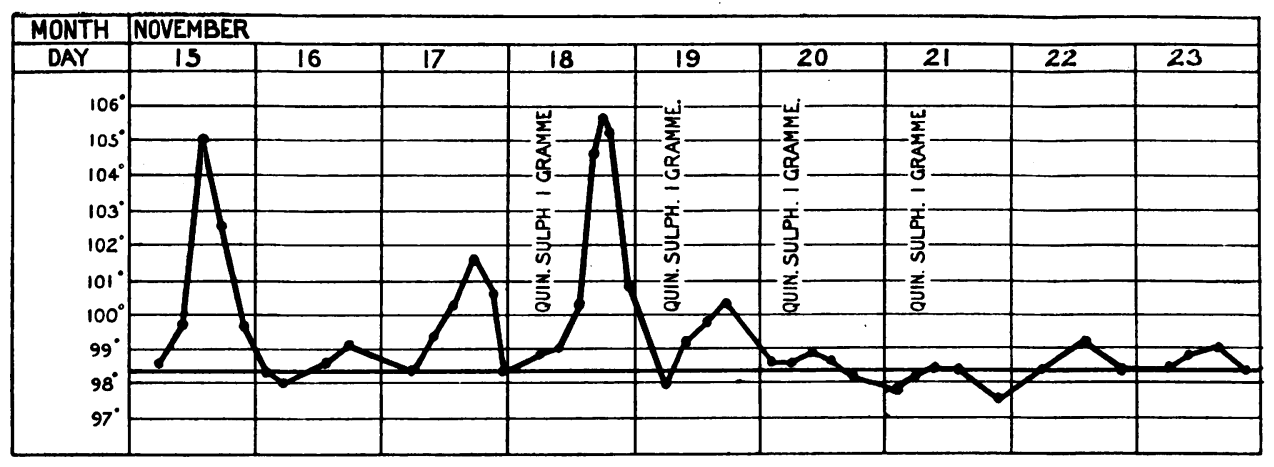

CASE 3.-F. Martinez Romano.

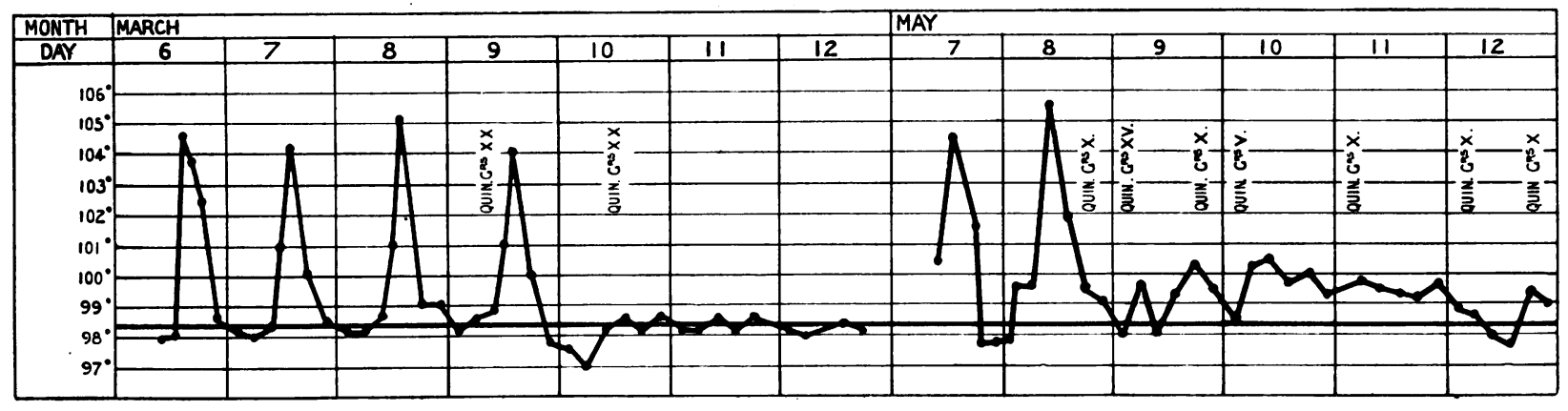

CASE 2.-Josè Pautiga.

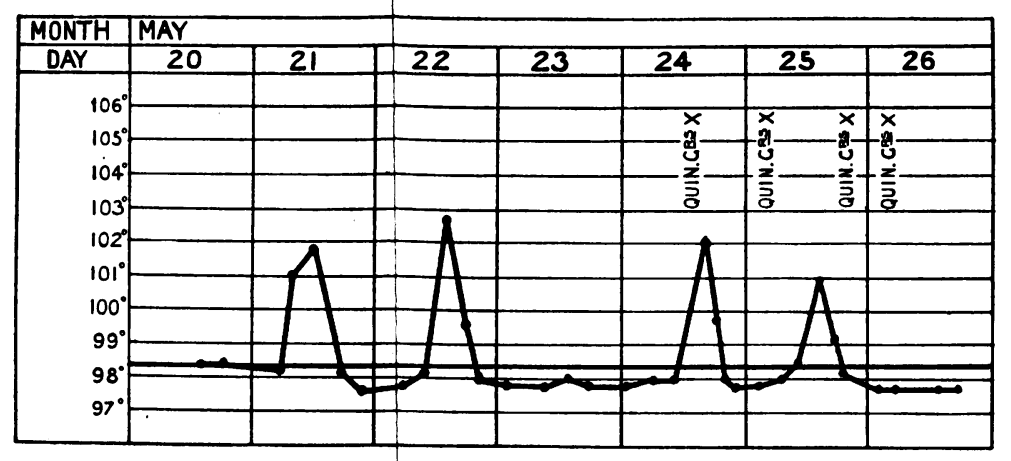

CASE 4.-M. S. Sanchez.

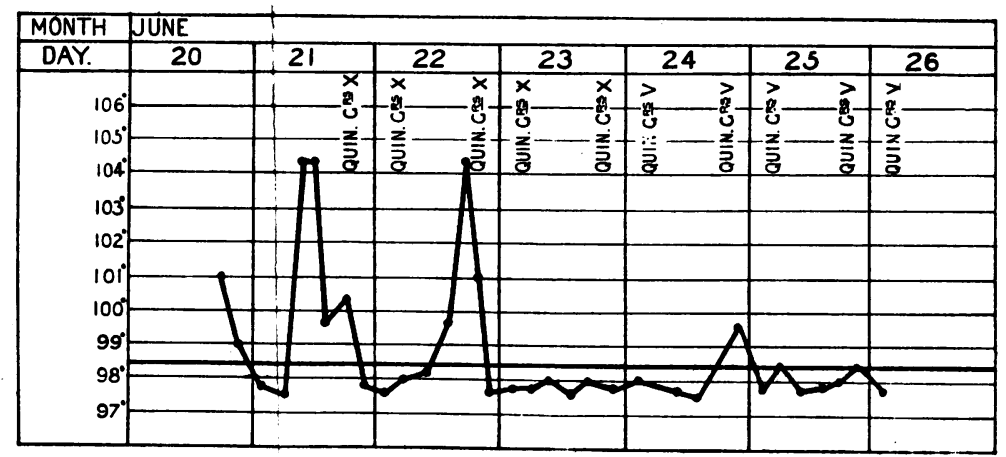

CASE 5.-E. Ameijeiras.

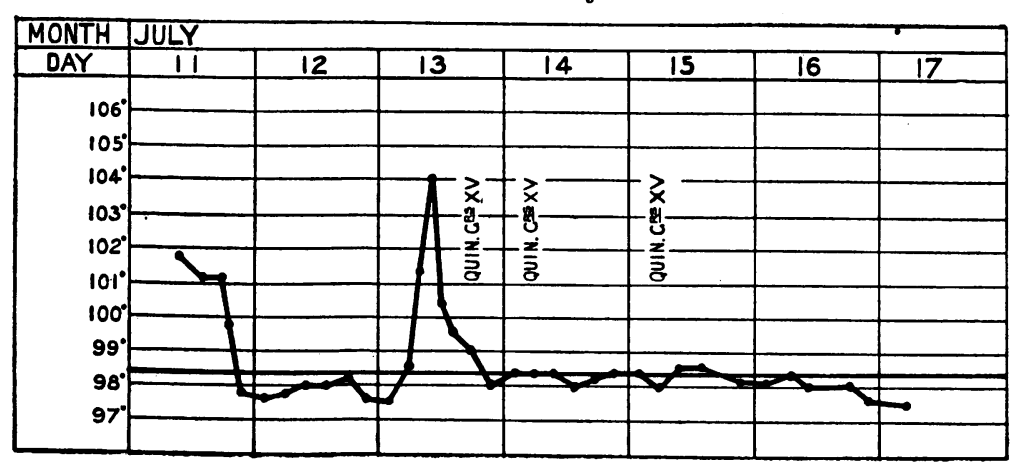

CaSE 6.-F. Nunez Rodrigues.

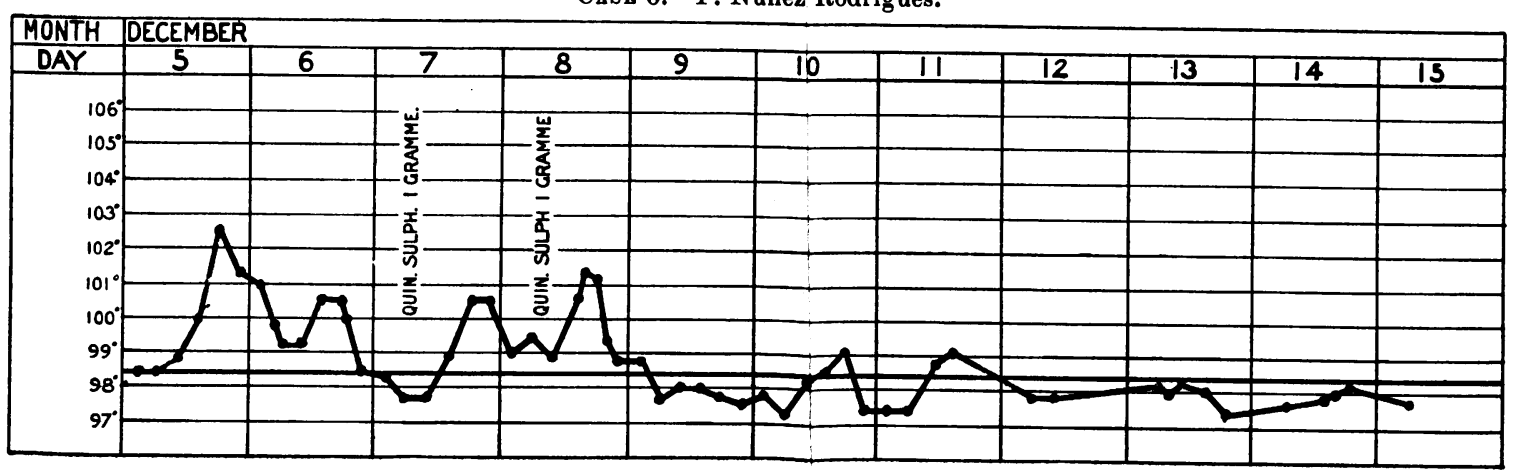


CaSe 7.-Juan Garrida Martin.

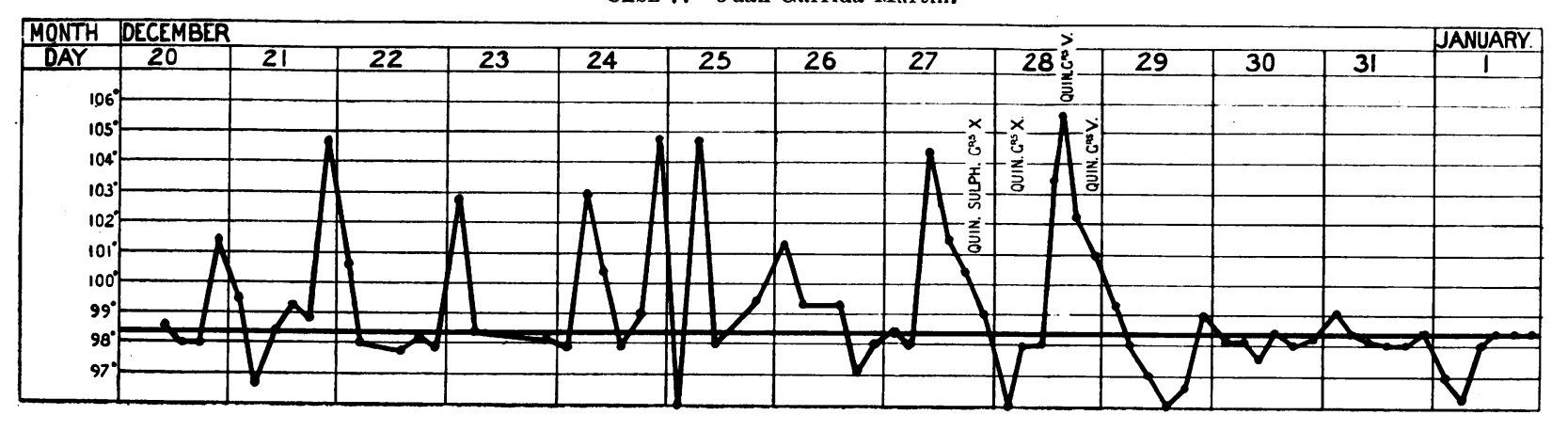

CASE_9.-Manuel Trigo Fernandez.

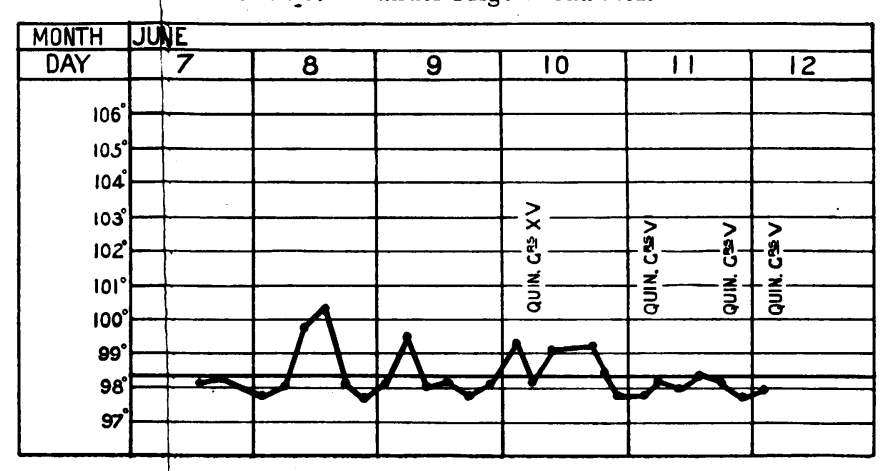

CASE 8.-Josè:Sanchez Concession.

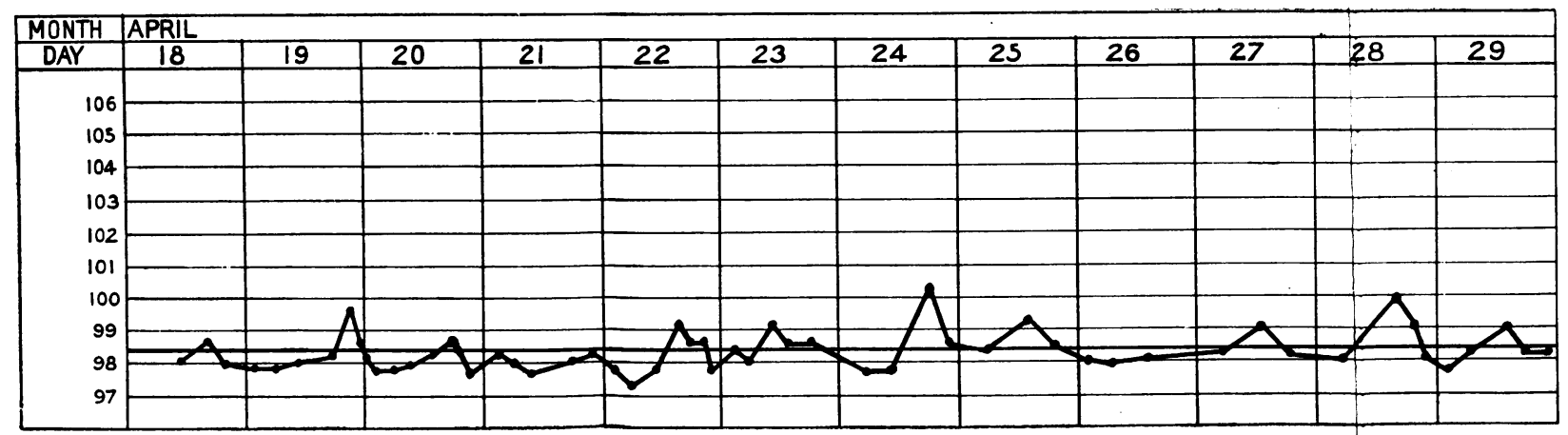

